\title{
Single-Implant Segmental Osteotomy for Repositioning of a Malpositioned Endosseous Implant: A Case Report
}

\author{
Somoza-Martín J M', García-García A , Gallas- \\ Torreira $\mathrm{M}^{2}$, Blanes-Vázquez $\mathrm{S}^{3}$ and Pérez-Sayáns \\ $\mathbf{M}^{4 *}$ \\ ${ }^{1}$ Department of Oral Surgery, School of Dentistry, \\ University of Santiago de Compostela, Spain \\ ${ }^{2}$ Comprehensive Adult Dental Care, School of Dentistry, \\ University of Santiago de Compostela, Spain \\ ${ }^{3}$ School of Dentistry, University of Santiago de \\ Compostela, Spain \\ ${ }^{4}$ Oral Surgery and Implantology Unit, Instituto de \\ Investigación Sanitaria de Santiago (IDIS), Santiago de \\ Compostela, Spain \\ *Correspondling author: Pérez-Sayáns M, Oral \\ Surgery and Implantology Unit, Faculty of Medicine and \\ Dentistry, Instituto de Investigación Sanitaria de Santiago \\ (IDIS), Santiago de Compostela, Entrerríos s/n, Santiago \\ de Compostela C.P. 15782, Spain
}

Received: April 25, 2017; Accepted: May 29, 2017;

Published: J une 12, 2017

\begin{abstract}
Single-implant segmental osteotomy consists of osteotomy of a bone segment encasing the implant and its relocation in a more favourable position. This case report describes the repositioning of a malpositioned endosseous implant by means of single-implant segmental osteotomy. The patient was a young woman with a malpositioned implant in the anterior sector, leading to poor aesthetics. Segmental osteotomy is a safe, cost-effective and predictable option for resolving problems of this type, offering excellent clinical and aesthetic outcomes.
\end{abstract}

Keywords: Endosseous implants; Segmental osteotomy; Malpositioned implants

\section{Introduction}

In recent years, osseointegrated implants have become the treatment of choice for dental prostheses. The treatment aims not only to achieve correct osseointegration, but also good aesthetics, particularly in the anterior sector. This requires that the implant be correctly aligned in all three spatial dimensions [1,2]. Misalignment, whether due to poor planning or poor technique, will in most cases result in poor aesthetics, and will often result in poor functionality. Rather few options are available for correcting a malpositioned implant. One possibility is single-implant segmental osteotomy, consisting of osteotomy of a bone segment encasing the implant, and its subsequent relocation in a more favourable position [37]. This technique is based on single-tooth segmental osteotomy, used to correct malpositioned dental units that cannot be treated orthodontically $[8,9]$, and typically involving one or various teeth that are either ankylosed or have undergone supraeruption. Like ankylosed teeth, endosseous dental implants have no periodontal ligament and thus cannot be repositioned orthodontically.

In this case report we described a single-implant segmental osteotomy in the anterior maxillary, to correct a malpositioned endosseous implant with poor aesthetics.

\section{Case Presentation}

A healthy 24-year-old female presented in our service with an implant-supported rehabilitation of the upper lateral incisor, with poor aesthetics that the patient was concerned about and wanted corrected. The implant was a Calcitek implant with hydroxyapatite surface, $13 \mathrm{~mm}$ long and $3.25 \mathrm{~mm}$ in diameter (Sulzer Calcitek Inc., Carlsbad, CA, USA), placed 4 years previously. Since placement, three different crowns had been fitted in an attempt to improve aesthetics,

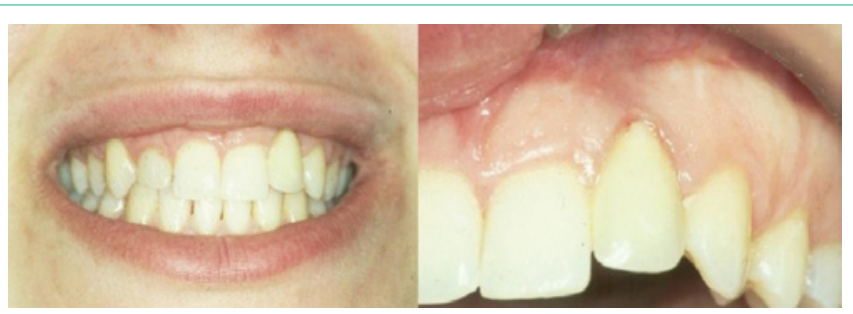

Figure 1: Intraoral photograph shows the excessively long crown (tooth position 22) before treatment. The aesthetic problem was particularly significant given the patient's gingival smile.

without success.

Intraoral examination indicated a long crown with gingival margin located about $3 \mathrm{~mm}$ above its ideal position and showing inflammation. The aesthetic problem was evident because the patient has a gingival smile (Figure 1). After detailed clinical and radiological study, we concluded that the implant was incorrectly positioned: too vestibular, slightly too apical, and excessively angled. The implant was well-integrated and did not show other problems. The treatment options were discussed with the patient and a decision was made to perform a single-implant segmental osteotomy.

Before surgery, maxillary and mandibular impressions were obtained, and cast surgery was performed, indicating that the implant segment needed to be repositioned $3 \mathrm{~mm}$ lingually and 1 $\mathrm{mm}$ coronally. Likewise before surgery, the prosthetic crown was removed, and the implant capped with a temporary capping screw. A provisional adhesive fixed prosthesis was made for attachment to the adjacent teeth.

Surgery was performed under local infiltration anaesthesia with
Austin J Dent - Volume 4 Issue 5 - 2017

ISSN : 2381-9189 | www.austinpublishing group.com

Pérez-Sayáns et al. @ All rights are reserved
Citation: Somoza-Martín JM, García-García A, Gallas-Torreira M, Blanes-Vázquez S and Pérez-Sayáns M. Single-Implant Segmental Osteotomy for Repositioning of a Malpositioned Endosseous Implant: A Case Report. Austin J Dent. 2017; 4(5): 1082 


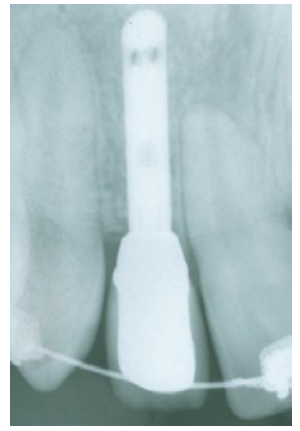

Figure 2: Pre-surgery periapical radiograph showing the closeness of the implant to the adjacent teeth.

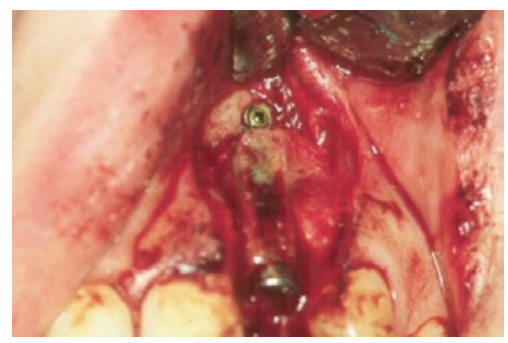

Figure 3: The bone segment moved to the correct position and immobilized with a mini-plate and two micro-screws in its apical part.

4\% articaine containing 1:200,000 epinephrine. A trapezoidal flap was raised by an incision marginal to the implant and two lateral incisions. This gave excellent access to the outer cortex of the maxillary in the region of the implant. Based on our experience in alveolar distraction osteogenesis [10], we did not detach the palatal mucoperiosteum, in order to maintain vascularization of the freed bone segment. The bone segment was freed by three osteotomies, one mesial, another distal, and another apical to the implant. Since the distance between the implant and the adjacent teeth was only $2 \mathrm{~mm}$ (Figure 2), the osteotomies were performed manually with the aid of chisels. The lingual osteotomy proved difficult, but we eventually managed to fully free the segment, pedicled to the palatal muciperiosteum, in which we made two small incisions to give greater mobility. We then re-positioned the segment in the required position, and fixed its apical region with a mini-plate and two micro-screws from an alveolar distraction system (LEAD System, Leibinger, Kalamazoo, MI, USA) (Figure 3).

To achieve the best possible aesthetic result, we increased vestibular volume in the region of the implant by means of guided bone regeneration, using Bio-Oss deproteinized bovine bone (Geistlich Pharma AG, Wolhusen, Switzerland) and a collagen membrane (Bio-Gide, Geistlich Pharma AG, Wolhusen, Switzerland). The mucoperiosteal flap was closed and sutured with 4-0 silk, leaving the implant submerged. Treatment with antibiotics, anti-inflammatories and chlorhexidine mouthrinse was prescribed.

Twenty-four hours later the patient returned for check-up, showing oedema in the left facial area, together with facial and intraoral haematoma, but without reporting pain. The adjacent teeth maintained vitality.



Figure 4: Photograph showing the patient's smile after the treatment, showing pleasing aesthetics with normal dimensions of the crown of tooth 22 and presence of papillae.

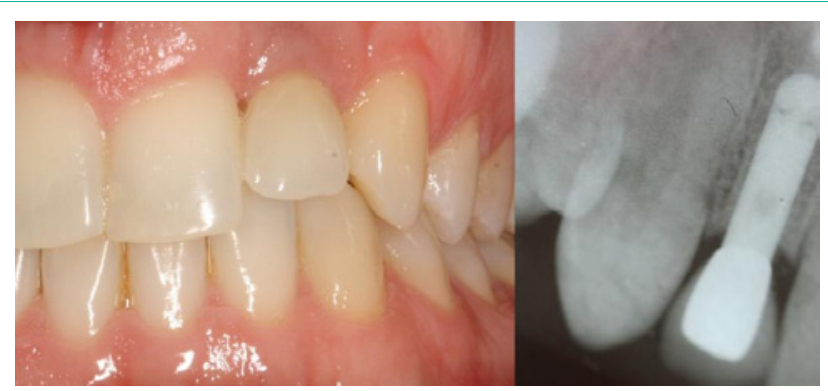

Figure 5: 12 Years post-operative view and radiographical follow-up.

At 4 months a second intervention was performed. Perfect integration of the bone segment was observed at this time. An impression were obtained and a screw-retained metal crown was made. The new position of the implant enabled anatomically adapted crown size and shape, and recuperation of the gingival outline, with papillae and gingival margin at the same level as the contralateral tooth (Figure 4). The patient expressed full satisfaction with the final aesthetic result (Figure 5).

\section{Discussion}

Aesthetic problems due to malpositioning of an implant in the anterior sector are generally resolved by the use of angled abutments. However, this approach often leads to an increase in the unfavourable forces placed on the implant, to poor soft tissue emergence and contours, and/or to creation of areas that the patient cannot maintain properly [5]. In most cases, this type of treatment leads to unfavourable aesthetic results and in the long term to implant failure. In the case presented here, attempts had previously been made to resolve the problem with an angled abutment, but without achieving satisfactory results even after trying three different crowns. Currently, the different options available under these circumstances include:

1) Restoring the edentulous space by conventional means, i.e. leaving the implant submerged and without crown ("putting the implant to sleep"). This option means that the patient does not have to tolerate surgery for implant removal, but cannot otherwise be considered as a successful treatment, in that the implant remains malpositioned.

2) Removing the implant with a trephine burr, and replacing it with a new implant in the correct position at a later date, after regrowth of the bone [11]. This approach is fully effective, but is often not possible because insufficient bone is available around the existing 
implant for removal of the implant without causing fenestration.

3) Retrieving the implant and surrounding bone with a hollow trephine burr, and re-positioning it correctly. The main drawback of this approach is that the bone graft is a free graft, not vascularized, with correspondingly higher risk of complications.

4) Distraction osteogenesis, with the implant included within the transport segment. In our opinion this is probably the treatment of choice when resolution of the problem requires movement of large bone blocks containing several implants, or in cases requiring movement of the bone block over a long distance, as described by Zechner [12].

5) Single-implant segmental osteotomy and subsequent repositioning of the implant.

Single-implant segmental osteotomy has been reported several times in the literature [3-7] and acceptable long-term outcomes in terms of function and aesthetic have been reported previously [13]. This single-stage surgical technique may be a treatment option that should be considered when faced with a malpositioned implant in the anterior region, where it is important to obtain optimal aesthetics, and where the surgeon's room for manoeuvre is very limited because of limited bone availability in this region and because of the close presence of adjacent teeth that restrict access. In addition, correct implementation of this technique in our opinion requires maintenance of vascularization of the bone segment, to favour its integration into the adjacent bone, and above all to achieve proper cicatrization of soft tissues, which is critical for good aesthetics.

In conclusion, the resolution of implant misalignment requires careful consideration of the available options, and careful planning of each particular case. Close coordination between the different professionals involved in the procedure is essential.

\section{References}

1. Tarnow DP, Cho SC, Wallace SS. The effect of inter-implant distance on the height of inter-implant bone crest. J Periodontol. 2000; 71: 546-549.

2. Buser D, Martin W, Belser U. Optimizing Esthetics for Implant Restorations in the Anterior Maxilla: Anatomic and Surgical Considerations. J of Oral Maxillofac Implants. 2004; 19: 43-61.

3. Svensson B, Adell R, Swartz B. Correction of implant malalignment by segmental osteotomy: a case report. Int J Oral Maxillofac Implants. 1993; 8: $459-463$.

4. Martin RJ, Goupil MT, Goldschmidt M. Single-implant segmental osteotomy: a case report. Int J Oral Maxillofac Implants. 1998; 13: 710-712.

5. Storum K, Carrick JL. Implant-osseous osteotomy for correction of the misaligned anterior maxillary implant. Dent Clin North Am. 2001; 45: 181-187.

6. Kassolis JD, Baer ML, Reynolds MA. The segmental osteotomy in the management of malposed implants: a case report and literature review. J Periodontol. 2003; 74: 529-536.

7. Raghoebar GM, Visser A, Vissink A. Correction of a malpositioned endosseous implant by a segmental osteotomy: a case report. Int J Oral Maxillofac Implants. 2005; 20: 627-631.

8. Burk JL, Provencher RF, McKean TW. Small segmental and unitooth osteotomies to correct dentoalveolar deformities. J Oral Surg. 1977; 35: 453460.

9. Kaminishi RM. Segmental osteotomies to optimize restorative procedures. Oral Maxillofac Clin North Am. 1994; 6: 671-678.

10. García A, Somoza JM, Gándara P, López J. Minor-complications arising in alveolar distraction osteogenesis. J Oral Maxillofac Surg 2002; 60:496-501.

11. Watanabe F, Hata Y, Mataga I, Yoshie S. Retrieval and replacement of a malpositioned dental implant: a clinical report. J Prosthet Dent. 2002; 88: 255-258.

12. Zechner W, Bernhart T, Zauza K, Celar A, Watzek G. Multidimensional osteodistraction for correction of implant malposition in edentulous segments. Clin Oral Implants Res. 2001; 12: 531-538.
Austin J Dent - Volume 4 Issue 5 - 2017

ISSN : 2381-9189 | www.austinpublishing group.com

Pérez-Sayáns et al. (C) All rights are reserved
Citation: Somoza-Martín JM, García-García A, Gallas-Torreira M, Blanes-Vázquez S and Pérez-Sayáns M. Single-Implant Segmental Osteotomy for Repositioning of a Malpositioned Endosseous Implant: A Case Report. Austin J Dent. 2017; 4(5): 1082. 